\title{
Comparative Steady State Cross-Over Bioequivalence Study of 35mg Trimetazidine Extended-Release Tablets
}

\author{
Ashish Shedage*, Abhishek Khanna, Milind Gole, Shrinivas Purandare and Geena Malhotra
}

Department of Clinical and Bioequivalence Research, Cipla Limited, R\&D, Center, Mumbai, India

\begin{abstract}
Background: Trimetazidine (TMZ), an anti-ischemic drug, protects the myocardial cell from the harmful effects of ischemia. This study is aimed to determine bioequivalence between the test (Trimetazidine ER Tablet of Cipla Limited, India) and the reference (Preductal MR Tablet of Servier, France) products at steady state in 24 healthy adult male volunteers under fed conditions.

Method: We conducted a randomized, open-label, balance, two-treatment, two-period, two-sequence, crossover steady state bioequivalence study separated by a washout period of 7 days. Participants were randomly assigned to receive $35 \mathrm{mg}$ of trimetazidine either test or reference products twice daily (12 hours interval) after standardized breakfast and dinner on day 1 to day 4 , followed by single dose on day 5 after breakfast in each study period. Post-dose blood samples were collected up to 36 hours and analyzed for trimetazidine using a validated LC-MS/MS method. Steady-state trimetazidine concentrations were statistically analyzed using SAS ${ }^{\circledR}$ software for windows (version 9.1).
\end{abstract}

Results: The $90 \%$ confidence intervals $(\mathrm{Cl})$ for trimetazidine $\left(\mathrm{AUC}_{\mathrm{Tau}}\right.$, and $\mathrm{C}_{\text {maxss }}$ ) were the conventional bioequivalence range of $80.00-125.00 \%$, thus permitting one to conclude for bioequivalence. Furthermore, both formulations were well tolerated and had no-serious adverse event reported.

Conclusion: The test and reference formulations of trimetazidine meet the regulatory criteria for bioequivalence both in terms of rate and extent of absorption.

Keywords: Trimetazidine; Steady state pharmacokinetics; Bioequivalence

\section{Introduction}

Trimetazidine dihydrochloride is a clinically effective, well tolerated anti-anginal agent which has been used in the prophylaxis and treatment of angina pectoris [1]. It also has been reported to be an effective agent for treating heart failure [2].The chemical structure of trimetazidine (TMZ) was shown in Figure 1. This drug acts via metabolic pathway by inhibition of the enzyme 3-ketoacyl coenzyme A thiolase, which results in a shift of cardiac metabolism from free fatty acid metabolism to glucose oxidation [3,4]. The cardiac utilization of glucose as a substrate for energy production is an effective approach to treat ischemic myocardium because it requires less oxygen consumption to produce the same amount of adenosine triphosphate [4,5]. Two formulations of trimetazidine are available in the clinical practice, the immediate-release and the modified-release tablet. The latter dosage form was developed to reduce frequency of dosing while maintaining sustained 24 hour coverage [6]. It has been shown to improve patient compliance thus more effective than a conventional tablet [7]. The pharmacokinetic profiles of two dosage forms of trimetazidine are different. After oral administration, both trimetazidine tablets are rapidly absorbed. The immediate-release tablet reached peak plasma concentration within 2 hours compared with 5 hours for the modified release tablet [8]. Trimetazidine is widely distributed throughout the body and mainly excreted in urine with approximately $60 \%$ eliminated unchanged. Eight metabolites of the drug have been detected in urine, but their properties were not known [1]. The elimination half-life of conventional tablet and modified-release tablet are approximately 6 and 7 hours, respectively [8].

Trimetazidine modified-release tablet has been manufactured and marketed by several pharmaceutical companies. Bioequivalence is a surrogate for therapeutic equivalence used to demonstrate whether two different drug products with the same active substance, strength and dosage form are comparable in terms of rate and extent of absorption. The present study aimed to compare bioavailability of two trimetazidine modified-release products in healthy male volunteers. The first one is a test product, Trimetazidine $35 \mathrm{mg}$ Extended Release (ER) Tablet manufactured by Cipla Limited, India and another one is reference product, Preductal MR Tablet manufactured by Servier, France.

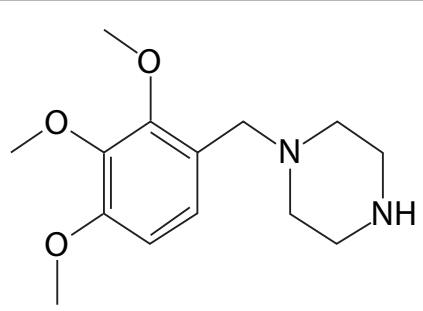

Figure 1: Trimetazidine Dihydrochloride

*Corresponding author: Ashish Shedage, Department of Clinical and Bioequivalence Research, Cipla Limited, R \&D, Center, LBS Marg, Vikhroli (W), Mumbai 400 083, India, Tel: +91 22 25756479; Fax: +91 22 25756355; E-mail ashish.shedge@cipla.com

Received September 10, 2014; Accepted October 13, 2014; Published November 01, 2014

Citation: Shedage A, Khanna A, Gole M, Purandare S, Malhotra G (2014) Comparative Steady State Cross-Over Bioequivalence Study of $35 \mathrm{mg}$ Trimetazidine Extended-Release Tablets. J Bioequiv Availab 6: 192-196. doi:10.4172/jbb.1000203

Copyright: @ 2014 Shedage A, et al. This is an open-access article distributed under the terms of the Creative Commons Attribution License, which permits unrestricted use, distribution, and reproduction in any medium, provided the original author and source are credited. 


\section{Subjects and Methods}

This study was conducted at the Manipal AcuNova KMC Clinical Pharmacology Unit (Mangalore, India) in accordance with the ethical standards laid down in the Declaration of Helsinki, the International Conference on Harmonization Good Clinical Practice Guidelines, and the note for guidance on the investigation of bioavailability and bioequivalence laid down by the European Agency for the Evaluation of Medicinal Products [9,10]. Prior to the start of the study, written informed consent was obtained from all subjects and the protocol was approved by the local ethical review boards.

\section{Subjects selection}

Healthy human subjects between 18 to 45 years of age, having Body Mass Index (BMI) between 18.5 and $24.9 \mathrm{~kg} / \mathrm{m}_{2}$ were included in this study. Eligible subjects were considered healthy, as evidenced by a lack of medical history of significant diseases and clinically significant abnormal findings during the pre-study screening, physical examination, chest X-ray, 12-lead ECG and laboratory investigations, including hematology, biochemistry, serology, and urine analysis. Breath alcohol test and test for drugs of abuse were negative at the time of screening. Hepatitis A, B, C and antibodies to HIV-I and II, VDRL were negative or non-reactive. Subjects were excluded if they were allergic to trimetazidine; had history or presence of cardiovascular, pulmonary, hepatic, renal, haematological, gastrointestinal, metabolic, immunologic, dermatologic, neurological or psychiatric disease; had participated in any other clinical investigation using investigational product or had donated or lost more than $350 \mathrm{~mL}$ of blood within 90 days prior to the screening of the study.

\section{Study design}

The pharmacokinetics of two trimetazidine extended release tablet formulations were compared in a randomized, open-label, balance, two-treatment, two-period, two-sequence, cross-over steady state bioequivalence study. The subjects were administered treatment in a crossover manner under fed conditions according to the randomization schedule. Steady state dosing was carried out for the reference formulation to determine the intra-subject variability of the reference formulation. Two periods of the study were separated by washout period of 7 days.

Prior to check-in of subjects into the clinical study facility and before first dosing, the subjects were given a detailed explanation of the aim, restrictions and possible side effects which could be experienced as a result from administration of a study drugs. Subjects gave their written consent to participate in the study by signing an informed consent form. Check-in was followed by catering, vital signs examination and well-being assessment on the pre-study day. There was a supervised overnight fasting period of at least 10 hours before dosing. Thereafter, all the subjects received either the test or reference product orally with $240 \mathrm{~mL}$ of water at room temperature twice daily (at 12 hours interval), 30 min after the start of standardized breakfast and dinner on day 1 to day 4 , followed by single dose on day 5 after breakfast.

Thereafter, a series of the blood samples were taken over the following 36.00 hour post-dose. Subjects were allowed to leave the Clinical Pharmacology Unit (CPU) after their vital signs examination are done and well-being was assessed after 36.00 hours post-dose blood sample was collected.

Subjects were not recruited in the study if they had taken any prescription medication or over-the-counter products, topical medication meant for systemic absorption within 14 days prior to administration of Investigational Product. All subjects were instructed to abstain from consuming grapefruit or its products and alcohol/ alcoholic products for at least 48 hours prior to dosing. They were also be instructed to abstain from consuming xanthine containing products (chocolate, tea, coffee or cola drink), smoking and consuming tobacco or tobacco containing products for at least 24 hours prior to dosing.

\section{Pharmacokinetic sampling}

Blood samples of $6 \mathrm{ml}$ were collected into labeled vacuum tubes containing $\mathrm{K}_{3}$ EDTA as anticoagulant. They were kept in an iced-water bath till they were centrifuged. The baseline pre-dose samples were collected immediately ( $2 \mathrm{~min}$ ) before dosing (morning and evening) on day 1 to 4 in each of the study period. On day 5 , the pre-dose sample was collected immediately ( $2 \mathrm{~min}$ ) before dosing in the morning and the post-dose samples were collected at $0.5,1.0,1.5,2.0,2.5,3.0,3.5$, $4.0,4.5,5.0,5.5,6.0,8.0,12.0,16.0,18.0,24.0$ and 36.0 hours. The first 15 blood samples on day 5 were collected from an ante-cubital vein using an indwelling cannula. The pre-dose samples on day 1, day 2, day 3 , day 4 and post dose samples on day 5 at 16.0, 18.0, 24.0 and 36.0 hours were collected by direct vein puncture.

The blood samples were centrifuged at $4^{\circ} \mathrm{C}$ and $3500 \mathrm{rpm}$ for 10 minutes. Each plasma sample was divided into two aliquots stored in separate, stoppered and suitably labelled tubes mentioning short study title, protocol number, subject number and time point. All plasma samples were stored in upright in a deep freezer set at $-70 \pm 5^{\circ} \mathrm{C}$ until analysis of the samples.

\section{Study outcomes}

The primary objective of this study was to compare the rate and extent of absorption of trimetazidine after administration of test or reference trimetazidine extended release tablet formulation in healthy adult human subjects in a randomized steady state bioequivalence study under fed conditions. The secondary objective of the study was to monitor the safety and tolerability of a multiple doses of trimetazidine $35 \mathrm{mg}$ extended release tablet, when administered to healthy adult human subjects under fed conditions. Safety was evaluated by monitoring all the subjects for adverse events throughout the course of the study. An Adverse Event (AE) was defined as any untoward medical occurrence in a subject or clinical investigation subject administered a pharmaceutical product, which need not necessarily have a causal relationship with the treatment. Recording of the vitals at pre-determined intervals and the comparison of pre and post study lab parameters formed the basis of the safety evaluation.

\section{Analytical assay}

The concentrations of trimetazidine in the plasma of the subjects were estimated using a validated LC-MS/MS method in accordance with the principles of Good Laboratory Practice (GLP). The validation criteria were system suitability, carry over test, specificity and selectivity, matrix effect, sensitivity, linearity (calibration curve), precision and accuracy, ruggedness, haemolysis effect, recovery, stability under different conditions, plasma dilution integrity and re-injection reproducibility. Quality control samples were distributed through each batch of study samples analyzed. To avoid bias, the analyst did not have access to the randomization code. Samples for any given subject for all time points were assayed under similar chromatographic conditions that were validated for the analysis of trimetazidine in human plasma. The lower limit of quantification (LLOQ) of this method for the estimation of trimetazidine concentrations in plasma was $2 \mathrm{ng} / \mathrm{ml}$. The 
linearity range was 2 to $150 \mathrm{ng} / \mathrm{mL}$ for trimetazidine. Concentrations below the LLOQ were set to 'zero' for pharmacokinetic and statistical calculations

\section{Pharmacokinetic assessment}

The statistical analysis were performed using SAS software (v. 9.1; SAS institute, Cary, NC). As per the requirements for steady state studies, the following parameters ware calculated individually for each subjects from trimetazidine plasma concentration: $\mathrm{AUC}_{\mathrm{Tau}}, \mathrm{C}_{\text {maxss' }}$, $\mathrm{C}_{\text {minss }}$ \% fluctuation.

Trimetazidine was considered for establishing bioequivalence between the test and reference product. The pharmacokinetic parameters $\mathrm{C}_{\text {maxss }}$ and $\mathrm{AUC}_{\mathrm{Tau}}$ were taken as primary efficacy variables.

All values below the limit of quantification were considered as zero for the computation of pharmacokinetic parameters and statistical calculations. The actual blood sampling time points were considered for the calculation of pharmacokinetic parameters.

\section{Statistical analysis}

Trimetazidine plasma concentration data and pharmacokinetic output from SAS software (v. 9.1; SAS institute, Cary, NC) were analyzed statistically. The statistical analysis was performed using SAS software (v. 9.1; SAS institute, Cary, NC).

Analysis of variance (ANOVA) was performed $(\alpha=0.05)$ on the $\log$ transformed pharmacokinetic parameters $\mathrm{C}_{\text {maxss }}$ and $\mathrm{AUC}_{\mathrm{Tau}}$ were analyzed using GLM ANOVA model with treatment as the main effect, period and sequence as fixed effects and subject nested within sequence as random effect.

Each analysis of variance also included calculation of least-square means, adjusted differences between formulation means and the standard error associated with these differences. The significance of the sequence effect was tested using the subjects nested within the sequence as the error term.

Geometric least-square mean (LSM) values were reported for log transformed $\mathrm{C}_{\text {maxss }}$ and $\mathrm{AUC}_{\mathrm{Tau}}$ Ratios of means were calculated using the LSM for $\log$ transformed $\mathrm{C}_{\text {maxss }}$ and $\mathrm{AUC}_{\mathrm{Tu}}$ Ratios of means were expressed as a percentage of the LSM for the reference treatment (Preductal MR Tablet).

Consistent with the two one-sided tests for bioequivalence, $90 \%$ confidence intervals for the difference between treatments, leastsquare means were calculated for $\log$ transformed $\mathrm{C}_{\text {maxss }}$ and $\mathrm{AUC}_{\text {Tau }}$. The confidence intervals were expressed as a percentage relative to the LSM of the reference treatments. The test product was to be considered as bioequivalent to the reference product, if the $90 \%$ CIs of $\mathrm{C}_{\text {maxss }}$ and $\mathrm{AUC}_{\mathrm{Tau}}$ of trimetazidine were within the acceptance range of $80-125 \%$.

\section{Results}

\section{Demographic data}

Of the 24 healthy male volunteers originally enrolled as a consequence, there were 24 subjects included in the statistical analysis of pharmacokinetics parameters. For demographic data, mean \pm SD of age, weight, height and BMI were $21.0 \pm 2.29$ years (range $18-27$ years), $58.472 \pm 7.0405 \mathrm{~kg}$ (range 48-78 kg), $168.86 \pm 6.619 \mathrm{~cm}$ (range 160$183 \mathrm{~cm}$ ) and $20.48 \pm 1.877 \mathrm{~kg} / \mathrm{m}^{2}$ (range $18.6-24.7 \mathrm{~kg} / \mathrm{m}^{2}$ ), respectively (Table 1). The laboratory data of all subjects were within an acceptable range. None was withdrawn from this study.

\section{Pharmacokinetic parameters and bioequivalence analysis}

The geometric mean plasma concentration-time profile after oral administration of Trimetazidine $35 \mathrm{mg}$ extended release $(\mathrm{T})$ and Preductal MR (R) under fed state were shown in Figure 2 respectively.

In the cross-over steady state study, it was observed that the mean peak-through fluctuation for trimetazidine was $94.697 \%$ for the test product and $98.289 \%$ for the reference product respectively (Table 2).

We observed the mean peak-through fluctuation in plasma trimetazidine concentration to be significantly higher than those reported literature.

Barre et al. [11] reported a fluctuation in trimetazidine concentrations of approximately $90.4 \%$ with the trimetazidine MR formulation at steady state. Onay-Besikci et al. [12] evaluated pharmacokinetic profile of trimetazidine and reviewed before the development of a modified-release (MR) formulation aimed to maintain the therapeutic plasma concentration with less fluctuation, a higher $\mathrm{C}_{\min }$ compared to immediate release from $(20 \mathrm{mg} \times 3)$ and only twice-daily application $(35 \mathrm{mg} \times 2)$.

In all the published studies, the dosing frequency was as per the labeling of the innovator product. In the multiple-dose study, twicedaily administration of the trimetazidine ER tablet was an ideal way to reach steady state, with less fluctuation. However, once daily administration of trimetazidine probably resulted in wide fluctuation in this study.

The intra subject variability of $\mathrm{AUC}_{\text {Tаu }}(\mathrm{ng} . \mathrm{hr} / \mathrm{mL})$ for trimetazidine was $11.994 \%$. Considering this result the sample size of 24 subjects was sufficient in order to conclude bioequivalence with the power of $80 \%$ at the $5 \%$ nominal level.

The mean plasma concentrations of trimetazidine were similar and yielded overlapping curves. This observation was supported by the $90 \%$ confidence intervals for the "test/reference" mean ratios of the ln-transformed pharmacokinetic variables $\mathrm{C}_{\text {maxss }}$ and $\mathrm{AUC}_{\mathrm{Tau}}$ which fell within the conventional bioequivalence range of $80 \%$ to $125 \%$. The formulation effects for the pharmacokinetic variables $C_{\text {maxss }}$ and $\mathrm{AUC}_{\mathrm{Ta \mu}}$ were statistically insignificant. Intra-subject variability observed for test and reference formulations was similar for all these two pharmacokinetic variables (Table 3).

A considerable period/treatment effect for $\mathrm{C}_{\text {maxss }}$ and $\mathrm{C}_{\text {minss }}$ for trimetazidine was observed in the fed steady state study. This effect might reflect the difference in the formulations, but it did not seem to have any impact on the study outcome because the confidence intervals for the log-transformed pharmacokinetic parameter fell within the acceptance range. Thus, the treatment effect can be ignored.

\section{Safety}

Both the formulations were well tolerated. No serious clinical

\begin{tabular}{|c|c|c|}
\hline & Mean $\mathbf{( \pm \mathbf { S D } ) \text { Value }}$ & Range \\
\hline Age (Years) & $21.0 \pm 2.29$ & $18-27$ \\
\hline Weight $\mathbf{( K g )}$ & $58.472 \pm 7.0405$ & $48-78$ \\
\hline Height $\mathbf{( c m )}$ & $168.86 \pm 6.619$ & $160-183$ \\
\hline BMI (kg/m $\left.\mathbf{m}^{\mathbf{2}}\right)$ & $20.48 \pm 1.877$ & $18.6-24.7$ \\
\hline Gender & \multicolumn{2}{|c}{ Male } \\
\hline Race & Asian \\
\hline
\end{tabular}

Table 1: Demographic data for trimetazidine bioequivalence study in 24 subjects. 


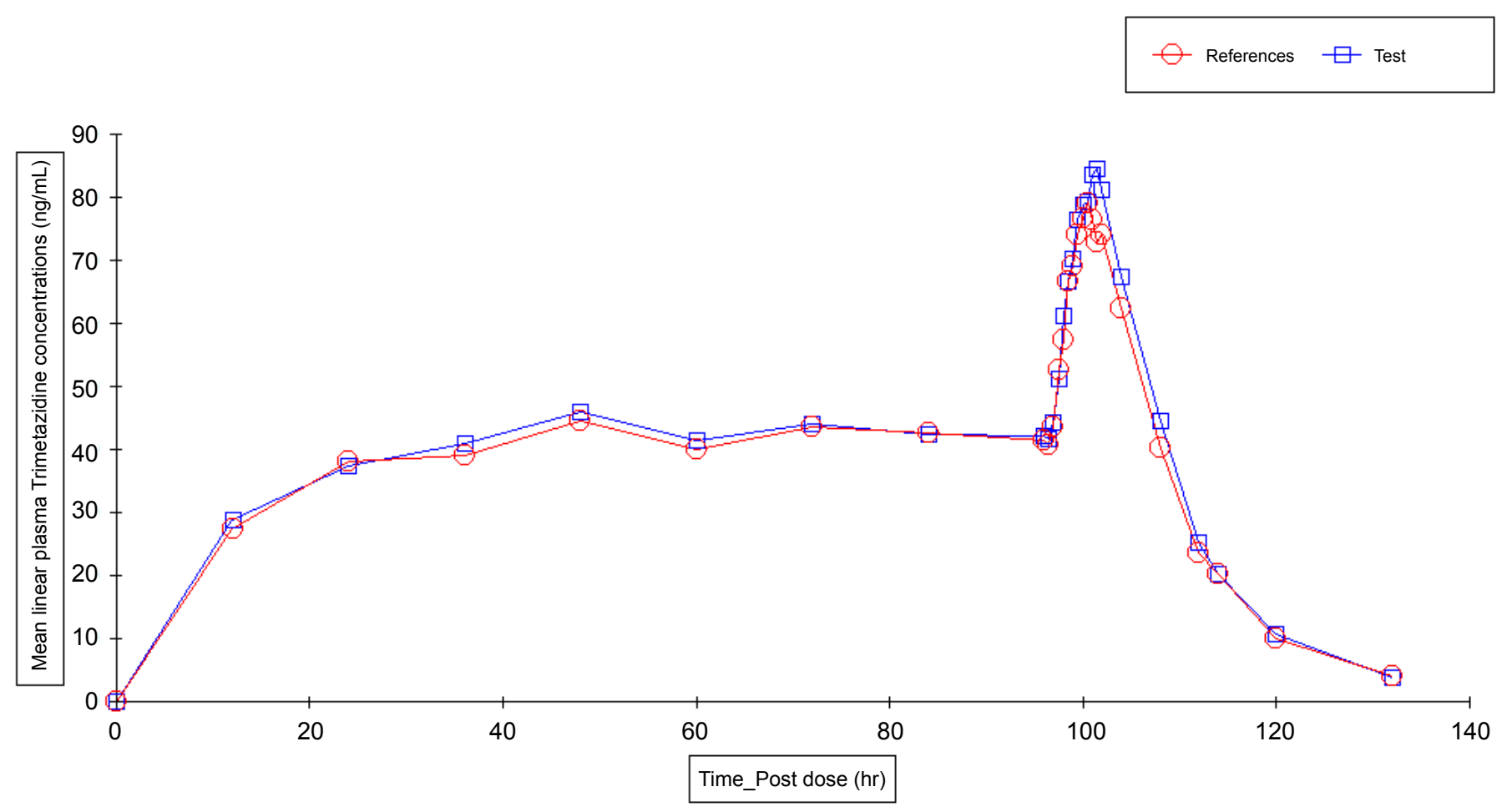

Test $=$ Trimetazidine ER Tablet of Cipla Limited, India Reference $=$ Preductal MR Tablet of Servier, France.

Figure 2: Comparative mean linear plasma concentration of trimetazidine.

\begin{tabular}{|c|c|c|c|}
\hline Formulation & Parameters & Test Product & Reference Product \\
\hline \multirow{3}{*}{ Trimetazidine } & $\mathbf{C}_{\text {maxss }}$ ( $\left.\mathbf{n g} / \mathbf{m L}\right)$ & $95.263 \pm 20.773$ & $90.794 \pm 23.973$ \\
\cline { 2 - 4 } & $\mathbf{C}_{\text {minss }}(\mathbf{n g} / \mathbf{m L})$ & $36.842 \pm 12.222$ & $34.376 \pm 15.102$ \\
\cline { 2 - 4 } & AUC $_{\text {Tuu }}(\mathbf{n g} \cdot \mathbf{h r} / \mathbf{m L})$ & $772.445 \pm 200.860$ & $725.606 \pm 201.223$ \\
\cline { 2 - 4 } & Fluctuation \% & $94.697 \pm 25.857$ & $98.289 \pm 40.257$ \\
\hline
\end{tabular}

Note: Data are shown as least square mean \pm standard deviation for pharmacokinetic parameters.

Abbreviations: $\mathrm{AUC}_{\mathrm{Tau}}$, area under curve at steady state; $\mathrm{C}_{\text {maxss }}$, peak concentration at steady state; $\mathrm{C}_{\text {minss }}$, minimum concentration at steady state

Table 2: Mean Pharmacokinetic Parameters of Trimetazidine at steady state.

\begin{tabular}{|c|c|c|c|c|c|c|}
\hline \multirow{2}{*}{ Analyte } & \multirow{2}{*}{ Study } & \multirow{2}{*}{ Parameters } & \multirow{2}{*}{$\%$ ratio $T / R$} & \multirow{2}{*}{$\%$ intra-CV } & \multicolumn{2}{|c|}{$90 \% \mathrm{Cl}$ for log-transformed data } \\
\hline & & & & & Lower Limit & Upper limit \\
\hline \multirow{2}{*}{ Trimetazidine } & \multirow{2}{*}{ Cross-over steady state study } & $\mathrm{C}_{\operatorname{maxss}}(\mathrm{ng} / \mathrm{mL})$ & 106.06 & 15.177 & 98.42 & 114.30 \\
\hline & & $\mathrm{AUC}_{\text {Tаu }}(\mathrm{ng} \cdot \mathrm{hr} / \mathrm{mL})$ & 106.93 & 11.994 & 100.78 & 113.45 \\
\hline
\end{tabular}

Abbreviations: $\mathrm{AUC}_{\mathrm{Tau}}$, area under curve at steady state; $\mathrm{C}_{\text {maxss }}$, peak concentration at steady state; $\mathrm{CV}$, coefficient of variation; $\mathrm{T} / \mathrm{R}$, test product/reference product; $\mathrm{Cl}$ confidence interval.

Table 3: Geometric mean ratio, intra subject variability, and $90 \%$ confidence intervals for trimetazidine

adverse events causing death, disability, hospitalization or dropout of the subjects were encountered. A reported mild adverse event, possibly related to treatment, was giddiness ( $4.16 \%$ for reference product). Post study clinical lab tests revealed normal results.

\section{Discussion}

Trimetazidine is a metabolic agent which exerts anti-ischaemic properties which have been widely used for treating myocardial ischemia and angina pectoris in patients with coronary artery disease [13-15]. TMZ, the first 3-ketoacyl coenzyme A thiolase inhibitor, reduces long-chain fatty acid oxidation resulting in a shift towards glucose oxidation during cellular ischemia. This results in an improved coupling of glycolysis with glucose oxidation, which has been shown to protect the ischemic heart [16].

TMZ has been marketed for over twenty years as a drink solution (Trimetazidine $20 \mathrm{mg} / \mathrm{ml}$ ) as well as an immediate release (IR) oral formulation (Trimetazidine $20 \mathrm{mg}$ tablets). Its indications cover (i) in cardiology: the prophylactic treatment of episodes of angina pectoris, (ii) in ophthalmology: the adjuvant treatment of the decline of visual acuity and visual field disturbances, presumably of vascular origin, and (iii) in ear-nose-throat pathology: the adjuvant symptomatic treatment of vertigo and tinnitus. The daily dose is $60 \mathrm{mg}$, taken in 3 doses.

In order to enhance patient compliance during long term 
treatment with TMZ, a modified release tablet, TMZ $35 \mathrm{mg}$, was developed. According to the regulatory guidelines [17], evidence has to be documented concerning rate and extent of drug absorption, fluctuation in drug concentrations, and variability in pharmacokinetics. The object of the present article is to describe some key steps taken in the characterization of the in vivo pharmacokinetics of the TMZ $35 \mathrm{mg}$ tablet (Trimetazidine Extended $35 \mathrm{mg}$ tablets) in comparison with the reference product.

The results of our study showed that these values of $\mathrm{AUC}_{\mathrm{Tau}}$ and $\mathrm{C}_{\text {maxss }}$ were all within the acceptable range in fed state. The power of the test was also calculated for $\mathrm{AUC}_{\text {Tau }}$ and $\mathrm{C}_{\text {maxs }}$. The powers of all parameters were above 0.80 which indicated that this study has adequate sample size.

Both trimetazidine modified-release products were well tolerated in the study population as there were only mild adverse events reported. Some limitations of the study design warrant mention. First, although the reference drug product is used for both male and female patients, all the subjects in the study were healthy male subjects. But this can be justified since no literature could be identified demonstrating that there is a gender effect for this drug related to pharmacokinetics.

It is important to maintain effective myocardial protection throughout a 24-hour period, especially in the early morning when the consequences of ischaemia are most severe [18]. To that respect and from sole pharmacokinetic considerations, one would anticipate the TMZ $35 \mathrm{mg}$ b.i.d. regimen to provide better patient protection. The clinical efficacy of TMZ ER $35 \mathrm{mg}$ has been currently confirmed in angina pectoris on exercise test performed 12 hours after the last intake of the drug. The time to $1 \mathrm{~mm}$ ST segment depression and time to angina were significantly delayed compared to placebo after 8-week treatment [18].

The results of the present study showed that the geometric mean ratios (90\% CIs) of the $\mathrm{AUC}_{\text {Tau }}$ and $\mathrm{C}_{\text {maxss }}$ of trimetazidine were $106.93 \%$ (100.78\%-113.45\%) and $106.06 \%$ (98.42\%-114.30\%), respectively. The values were within the acceptance range for bioequivalence.

In summary, the sustained release properties of the TMZ $35 \mathrm{mg}$ tablet have been demonstrated under steady state in healthy adult male subjects. These properties have been verified in humans where the new formulation, one tablet in the morning followed by one tablet in the evening, did provide similar total exposure, improved coverage over a 36-hour period as shown by the increased peak-trough fluctuation and no increase in inter-individual variability as compared to TMZ IR 20 mg tablet given three times a day. Furthermore, TMZ ER $35 \mathrm{mg}$ with a b.i.d. administration is likely to result in improved patient compliance and to provide better patient protection for the entire day.

\section{Conclusion}

The bioequivalence study of two modified-release tablets of $35 \mathrm{mg}$ trimetazidine in healthy male volunteers demonstrated pharmacokinetic equivalence in terms of both rate and extent of absorption.

\section{Acknowledgements}

We deeply thank the volunteers for their participation in this study.

\section{Disclosure}

This study was sponsored by Cipla Limited, Mumbai, India, and was conducted by Manipal Acu Nova KMC Clinical Pharmacology Unit, Mangalore, India. The authors report no conflicts of interest in this work.

\section{References}

1. McClellan KJ, Plosker GL (1999) Trimetazidine. A review of its use in stable angina pectoris and other coronary conditions. Drugs 58: 143-157.

2. Gao D, Ning N, Niu X, Hao G, Meng Z (2011) Trimetazidine: a meta-analysis of randomised controlled trials in heart failure. Heart 97: 278-286.

3. Kantor PF, Lucien A, Kozak R, Lopaschuk GD (2000) The antianginal drug trimetazidine shifts cardiac energy metabolism from fatty acid oxidation to glucose oxidation by inhibiting mitochondrial long-chain 3-ketoacyl coenzyme A thiolase. Circ Res 86: 580-588.

4. Vitale C, Marazzi G, Pelliccia F, Volterrani M, Cerquetani E, et al. (2011) Trimetazidine improves exercise performance in patients with peripheral arterial disease. Pharmacol Res 63: 278-283.

5. Lopaschuk GD, Stanley WC (1997) Glucose metabolism in the ischemic heart Circulation 95: 313-315.

6. Barré J, Ledudal P, Oosterhuis B, Brakenhoff JP, Wilkens G, et al. (2003) Pharmacokinetic profile of a modified release formulation of trimetazidine (TMZ MR $35 \mathrm{mg}$ ) in the elderly and patients with renal failure. Biopharm Drug Dispos 24: $159-164$

7. Gupta R, Sawhney JP, Narain VS (2005) Treatment of stable angina pectoris with trimetazidine modified release in Indian primary-care practice. Am J Cardiovasc Drugs 5: 325-329.

8. http://www.mims.com/Page.aspx?menuid=mng\&name=trimetazidine+hydroch loride $\& C T R Y=T H \& b r i e f=$ false\#Actions

9. World Medical Association (2008) Declaration of Helsinki; Ethical Principles fo Medical Research Involving Human Subjects. 59th World Medical Association General Assembly, Seoul.

10. The European Agency for the Evaluation of Medicinal Products (2001) Evaluation of Medicines for Human Use. Note for guidance on the investigation of bioavailability and bioequivalence.

11. Barré J, Ledudal P, Oosterhuis B, Brakenhoff JP, Wilkens G, et al. (2003) Pharmacokinetic profile of a modified release formulation of trimetazidine (TMZ MR $35 \mathrm{mg}$ ) in the elderly and patients with renal failure. Biopharm Drug Dispos 24: 159-164.

12. Onay-Besikci A, Ozkan SA (2008) Trimetazidine revisited: a comprehensive review of the pharmacological effects and analytical techniques for the determination of trimetazidine. CardiovascTher 26: 147-165.

13. Detry JM, Sellier P, Pennaforte S, Cokkinos D, Dargie H, et al. (1994) Trimetazidine: a new concept in the treatment of angina. Comparison with propranolol in patients with stable angina. Trimetazidine European Multicenter Study Group. Br J ClinPharmacol 37: 279-288.

14. Szwed H, Sadowski Z, Elikowski W, Koronkiewicz A, Mamcarz A, et al. (2001) Combination treatment in stable effort angina using trimetazidine and metoprolol: results of a randomized, double-blind, multicentre study (TRIMPOL II). TRIMetazidine in POLand. Eur Heart J 22: 2267-2274.

15. Manchanda SC, Krishnaswami S (1997) Combination treatment with trimetazidine and diltiazemin stable angina pectoris. Heart 78: 353-357.

16. Kantor PF, Lucien A, Kozak R, Lopaschuk GD (2000) The antianginal drug trimetazidine shifts cardiac energy metabolism from fatty acid oxidation to glucose oxidation by inhibiting mitochondrial long-chain 3-ketoacyl coenzyme A thiolase. Circ Res 86: 580-588.

17. CPMP/EWP/280/96 (1999) Note for guidance on modified release oral and transdermal dosage forms: Section II (Pharrnacokinetic and clinical evaluation). Adopted by EMEA (European Agency for Evaluation of Medicinal Products) London.

18. Génissel P, Chodjania Y, Demolis JL, Ragueneau I, Jaillon P (2004) Assessment of the sustained release properties of a new oral formulation of trimetazidine in pigs and dogs and confirmation in healthy human volunteers. Eur J Drug Metab Pharmacokinet 29: 61-68. 